\title{
Fault Diagnosis of Wind Turbine Based on ELMD and FCM
}

\author{
Xianjin Luo* and Xiumei Huang ${ }^{*}$
}

\author{
Department of Control and Computer Engineering, North China Electric Power University, Baoding 071003, China
}

\begin{abstract}
In view of failure characteristics of wind turbine gear box, this paper put forward a method for fault diagnosis based on the ensemble local means decomposition (ELMD) and fuzzy C-means clustering (FCM) method. By resolving the vibration signal of different fault state of high speed gear box by ELMD, the PF component was obtained with its singular value, which was composed of known sample followed by a test sample as the feature vector. The known sample was clustered by using the FCM clustering, and the test sample was recognized and classified . The experimental results show that the method for fault diagnosis based on ELMD and FCM clustering has good diagnosis results.
\end{abstract}

Keywords: ELMD, fault diagnosis, fuzzy C means clustering, gear box

\section{INTRODUCTION}

With the gradually decreasing cost of wind turbine manufacturing, wind power has become the preferred alternative energy [1, 2]. By the end of 2010, the installed capacity of China is $18,927.99 \mathrm{MW}$, while the cumulative installed capacity is $44,733.29 \mathrm{MW}$, which is highest in the world [3]. Wind turbines work in harsh environment for a long time, therefore with enhanced service life they are prone to enter the failure stage gradually. To detect the fault in wind turbine's efficiency and to maintain the safe operation have become an important function of wind power plants.

Fault diagnosis of wind power generator includes two aspects, which are; feature extraction and fault diagnosis. Fault feature is an important index to judge the running status of equipment. The method of extracting fault features is the key to improve the diagnosis accuracy $[4,5]$. The mechanical fault signal of wind turbine is a complicated time-varying and non-stationary signal and it is hard to process the fault signal by general frequency analysis methods. Studies [9-11] decomposed the original signal by using the method based on EMD decomposition, which overcomes the single correlation of frequency range and the sampling frequency and the lack of adaptability when using wavelet analysis. ELMD decomposition used in the [6] and EEMD decomposition used in the literature [8] can effectively inhibit the problem of mode mixing in the process of decomposition. Besides, in a study [7], the application of neural network in fault diagnosis achieved higher diagnostic accuracy, but its parameter adjustment was difficult and the convergence rate was slow, and in the study [8-13], the use of fuzzy C-means clustering achieved accurate rate with small training samples, which made the solution rapid and applicable.

Address correspondence to these authors at the Department of Control and Computer Engineering, North China Electric Power University, Baoding 071003, China; Tel: +8615131269311

E-mails: 13930854097@163.com and hairuo222@163.com
As an important part of wind turbine, the gear box is used to achieve the speed of the wind turbine rotor. Gear box is located in the engine room. The internal structure and the stress state are complex, therefore, its fault signal has the characteristics of being nonlinear and non-stationary. Based on this, the fault diagnosis method was used based on ELMD decomposition and FCM clustering in this paper. By calculating singular value of the PF component after EMD decomposition of fault vibration signal, the feature vector can be obtained, which is applied in clustering recognition of fault signal as the input of FCM clustering.

\section{ELMD METHOD}

Local mean decomposition (LMD) method decomposes a complex multi-component signals into the sum of a number of product signal functions where the instantaneous frequency is physically meaningful and a monotone function is as follows:

$x(t)=\sum_{i=1}^{K} P F_{i}(t)+u_{k}(t)$

Each PF component is the product of a pure frequency modulation signal and the envelope signal. Envelope signals represent the instantaneous amplitude of PF component.

Modal aliasing problem exists in the LMD decomposition and ensemble local mean decomposition (ELMD) method was proposed for its solution. First, a certain magnitude of white noise signal was provided to the target signal being decomposed. Following this, LMD method was used to decompose the target signal after adding white noise and the above process was repeated, with each added white noise signal being different. Finally, the mean of the respective multiple PF was decomposed as the final component of the decomposition. As the white noise will be evenly a "polluted" component of all different scales of the frequency space of the original signal, therefore, when a signal is added with white noise, it will be automatically decomposed into each passband of filter groups which are determined by white noise signal by LMD method. This will significantly reduce the modal aliasing. 
In summary, the ELMD decomposition process can be divided into the following steps:

Incorporation of a white noise signal to the target signal to obtain a mixed signal containing a white noise signal.

(2) Decomposition of the mixed signal by LMD to obtain a series of PF components and a residual component.

(3) Repetition of step (1) and (2).

(4) Overall averaging the PF components and residual components, to obtain the final decomposition results.

\section{FAULT DIAGNOSIS BASED ON ELMD AND FCM ALGORITHM}

\subsection{The Fuzzy c-Means Clustering Algorithm}

In 1973, Bezdek proposed the fuzzy C means clustering algorithm (FCM), as a kind of improved early hard c means clustering method. FCM algorithm divides $n$ vectors $x_{i}$ $(i=1,2, \ldots, n)$ into $c$ fuzzy groups, and calculates the clustering center of each group, so that the objective value function $J$ can be minimized. Element $u_{i j}$ represents the membership degree of the $i$ sample and $x_{i}$ belongs to the $j$ class. Membership degree matrix is $\boldsymbol{U}=\left[u_{i j}\right]$. To adapt to the introduction of fuzzy partition, the elements of membership matrix $\boldsymbol{U}$ are allowed to have a value between 0 and 1. Because of the normalization rules, the total membership degree of a data set is equal to 1 :

$\sum_{i=1}^{c} u_{i j}=1, \forall j=1, \ldots, n$

Objective value function expression is:

$J\left(U, c_{1}, \ldots, c_{c}\right)=\sum_{i=1}^{c} J_{i}=\sum_{i=1}^{c} \sum_{j}^{n} u_{i j}^{m} d_{i j}^{2}$

$0 \leq u_{i j} \leq 1 ; c$ is the number of cluster categories, $c_{i}$ is the clustering center of the fuzzy set $\mathrm{I} ; d_{i j}=\left\|c_{i}-x_{j}\right\|$ is the Euclidean distance between cluster center $i$ and the $j$ data points; and $m \in[1, \infty)$ is a weighted index. The necessary conditions of formula (4) to achieve minimal are as follows:

$c_{i}=\frac{\sum_{j=1}^{n} u_{i j}^{m} x_{j}}{\sum_{j=1}^{n} u_{i j}^{m}}$

and

$u_{i j}=\frac{1}{\sum_{k=1}^{c}\left(d_{i j} / d_{k j}\right)^{2 /(m-1)}}$

The above two equations are used to calculate the formula of the cluster center and membership degree matrix. Fuzzy c-means clustering algorithm is a simple iterative process. To determine the cluster centers $c_{i}$ and membership degree matrix $\boldsymbol{U}$, the following steps are followed:

Step 1: Using a random number between 0 and 1 to initialize the membership matrix $\boldsymbol{U}$, and to meet the constraint condition of type (2).

Step 2: Using formula (4) to calculate clustering center $c_{i}, i=1, \ldots, c$.

Step 3: Calculating the value function according to equation (3). If it is less than a determined threshold $\varepsilon$, the algorithm stops. The final membership degree of matrix $\boldsymbol{U}$ and cluster center $\boldsymbol{C}$ is obtained.

Step 4: If $J$ does not satisfy the conditions, the new $U$ matrix is calculated by (5), after which step 2 is followed.

Fuzzy diagnosis often uses the maximum membership principle or the principle of choosing the nearest to pattern recognition. In this paper the maximum membership principle was adopted.

\subsection{Gear Box Fault Diagnosis Process Based on ELMD and FCM Algorithm}

ELMD is an adaptive decomposition and can reflect the nature of the fault information. In the obtained PF components, different PF components contain different frequency components, with different fault information included in it. Therefore, by calculating the singular values of the PF components, the fault feature vector composed of singular values can be obtained.

Singular value decomposition has the same purpose as the eigenvalue decomposition, to extract the most important characteristics of a matrix. But the singular values of matrix have better stability, that is, when the matrix elements experience small change, the matrix singular value changes very little, while the matrix singular value also has scale invariance and rotation invariance. Therefore, it can effectively portray the characteristics of each PF component and the matrix consisting of fault feature vectors can effectively respond by demonstrating the characteristics of vibration signals [8].

The rolling bearing fault diagnosis steps are as follows:

(1) Extracting $N$ known vibration signals (including imbalance, bearing wear fault, gear wear fault) as known fault samples, with the other $M$ groups samples to be identified.

(2) Using ELMD method to decompose the vibration signals of rolling bearing and the singular values of $\mathrm{PF}$ components to compose the fault feature vector $\mathrm{x}_{\mathrm{i}}$. Following this, the standard sample $\boldsymbol{X}_{\mathbf{1}}=\left[x_{1}, x_{2}, \cdots, x_{N}\right]$ is obtained and the characteristics of the sample are identified $\boldsymbol{X}_{2}=\left[x_{1}, x_{2}, \cdots, x_{M}\right]$. Later, they are combined to form the diagnosis sample set $\boldsymbol{X}=\left[\boldsymbol{X}_{\mathbf{1}}, \boldsymbol{X}_{\mathbf{2}}\right]$.

The feature vector matrix $\boldsymbol{X}$ is standardized to get the initial membership matrix. Through standardization processing, the data is compressed in $[0,1]$ closed interval to obtain initial membership degree matrix $\widehat{\boldsymbol{X}}$. Standardized method is as follows:

$x_{i j}=\frac{x_{i j}-\min \left(x_{j}\right)}{\max \left(x_{j}\right)-\min \left(x_{j}\right)}$

The $\widehat{\boldsymbol{X}}$ is the input of FCM clustering. The fuzzy weighted number $m$ is set and the optimal range of $m$ is $[1.5,2.5]$. The number of cluster categories $c$ is determined and iteration stop threshold $\varepsilon$ is set. Using FCM clustering algorithm to cluster $\widehat{\boldsymbol{X}}$, after 
several iterations, the fault sample membership matrix $\boldsymbol{U}$ is obtained, where $u_{i j}$ represents the membership degree of the $j^{\text {th }}{ }^{\text {th }}$ sample for class $i_{-}{ }^{\text {th }}$.

\section{FAULT DIAGNOSIS OF GEAR BOX}

Wind turbine drive system mainly comprises of a main shaft, gear box, generator and coupling. Its main parts can be divided into shafts, bearings and gears. This paper used the vibration data of a $750 \mathrm{~kW}$ wind turbine transmission system for the analysis. This paper selected fault data based on three types of fault conditions as experimental data to make fault diagnosis. The three types of fault conditions were imbalance, bearing wear and gear wear of the high speed gear box. 4 sets of data were chosen in each fault condition with data sampling frequency of $4096 \mathrm{~Hz}$, and 3000 intercepts as sampling points in each set of data. Moreover, 3 groups of data were chosen out of 4 as known samples for clustering, and the remaining group was used to identify and diagnose as unknown sample.

\subsection{Fault Feature Extraction}

Taking the high speed gear box imbalance fault as an example, by using the ELMD decomposition, the white noise was affiliated whose amplitude was 0.1 (unit) and the ensemble average number was 50. Its time domain waveform and decomposition results are shown in Figs. (1, 2).

Decomposition results are shown in Fig. (2). After decomposition, five PF components and one residual component were obtained. PF5 indicated the periodic vibration of imbalance fault, as shown in the Fig. (2). No obvious mode mixing phenomenon appeared and its fault characteristic frequency could be obtained by calculating the envelope spectrum.

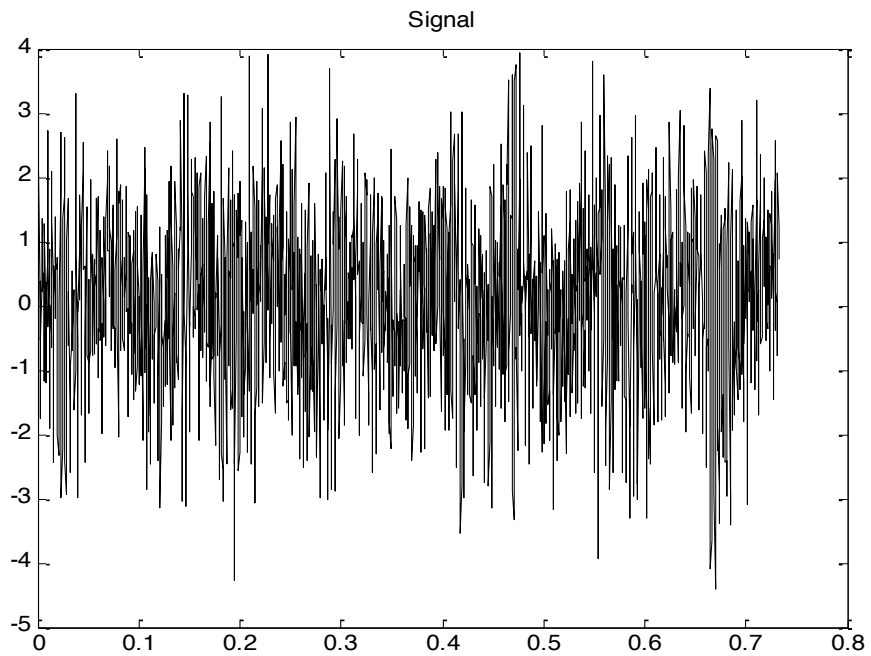

Fig. (1). The time domain waveform of Imbalance fault signal.

The singular value of PF components are as follows (Table 1):

Table 1. The singular value of PF components.

\begin{tabular}{|c|c|c|c|c|c|}
\hline PF & PF1 & PF2 & PF3 & PF4 & PF5 \\
\hline \hline Singular value S & 52.4969 & 33.3242 & 18.6817 & 14.6206 & 7.7715 \\
\hline
\end{tabular}

Taking the singular value as feature vector of the fault vibration signal.
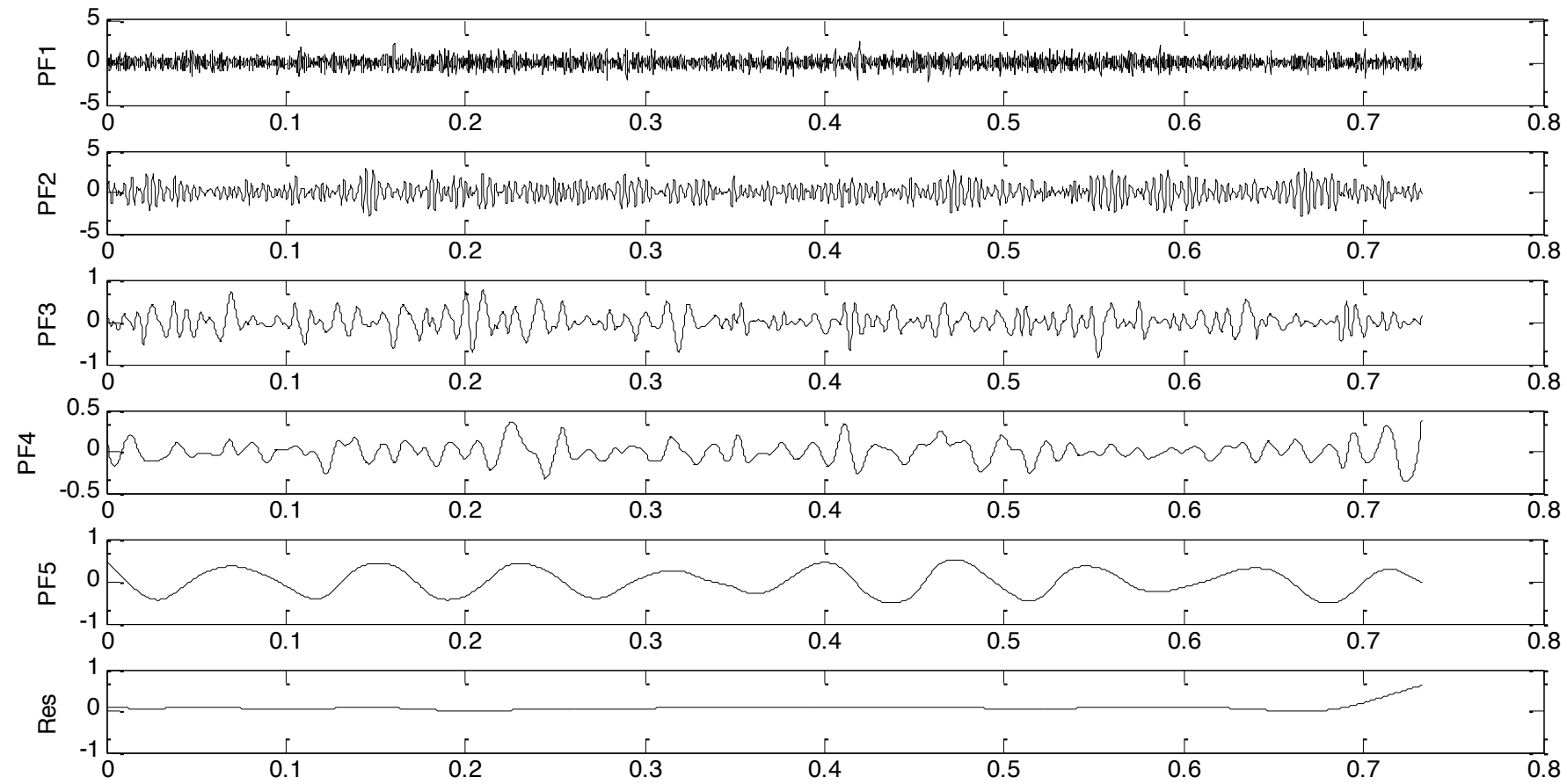

Fig. (2). The decomposition results of ELMD. 
Table 2. The feature vector of fault samples.

\begin{tabular}{|c|c|c|c|c|c|c|}
\hline Fault Types & Feature Vectors & PF1 & PF2 & PF3 & PF4 & PF5 \\
\hline Imbalance & $\begin{array}{l}x_{1} \\
x_{2} \\
x_{3} \\
x_{4}\end{array}$ & $\begin{array}{l}52.4969 \\
52.6388 \\
52.4969 \\
50.7029\end{array}$ & $\begin{array}{l}33.3242 \\
39.8600 \\
33.3242 \\
32.6208\end{array}$ & $\begin{array}{l}18.6817 \\
21.0683 \\
18.6817 \\
19.7934\end{array}$ & $\begin{array}{l}14.6206 \\
17.1704 \\
14.6206 \\
12.0941\end{array}$ & $\begin{array}{l}7.7715 \\
6.6120 \\
7.7715 \\
6.5669\end{array}$ \\
\hline Bearing Wear & $\begin{array}{l}x_{5} \\
x_{6} \\
x_{7} \\
x_{8}\end{array}$ & $\begin{array}{l}20.3159 \\
19.2563 \\
23.1940 \\
18.1611\end{array}$ & $\begin{array}{l}16.8637 \\
11.5555 \\
18.4435 \\
11.4021\end{array}$ & $\begin{array}{l}6.4024 \\
5.2730 \\
8.0359 \\
8.9889\end{array}$ & $\begin{array}{l}2.7301 \\
3.5942 \\
3.0189 \\
3.5529\end{array}$ & $\begin{array}{l}2.1696 \\
1.8105 \\
2.0692 \\
2.4200\end{array}$ \\
\hline $\begin{array}{l}\text { Gear } \\
\text { Wear }\end{array}$ & $\begin{array}{l}x_{9} \\
x_{10} \\
x_{11} \\
x_{12}\end{array}$ & $\begin{array}{l}83.8065 \\
73.8289 \\
79.8139 \\
74.5713\end{array}$ & $\begin{array}{l}32.9883 \\
31.6212 \\
31.3291 \\
29.8896\end{array}$ & $\begin{array}{l}21.3462 \\
17.8661 \\
18.9961 \\
16.0121\end{array}$ & $\begin{array}{l}8.0150 \\
8.8928 \\
11.8191 \\
7.9505\end{array}$ & $\begin{array}{l}6.1611 \\
8.2726 \\
7.3044 \\
6.2381\end{array}$ \\
\hline
\end{tabular}

\subsection{Clustering and Classification of Fault Samples}

According to the above method, the singular value of each fault sample with the feature vector in each fault condition was obtained. 3 sets of data among 4 were taken as known samples for clustering and the remainder group was used to diagnose the fault as unknown sample (Table 2).

The feature vector is composed of characteristic matrix $X=\left[x_{1}, x_{2}, x_{3}, x_{5}, x_{6}, x_{7}, x_{9}, x_{10}, x_{11}, x_{4}, x_{8}, x_{12}\right]$. After standard treatment, the initial membership matrix $\widehat{X}$ was obtained as the input of FCM clustering. In the process of FCM clustering, the weighted index $m$ was 2 , and threshold $\varepsilon=10^{-5}$. The known fault category $c$ was equal to 3 , obtaining the cluster membership matrix $\boldsymbol{U}$. The result of fault diagnosis is shown in Table 3 .

From Table 3, it can be observed that the diagnosis method of high speed gearbox for wind turbine based on ELMD and FCM clustering achieved good results. Besides, the classification of known samples was absolutely correct, the cluster membership was substantially larger than 0.9 and the three unknown samples were correctly classified.

\section{CONCLUSION}

For the three fault conditions of high speed gear box for wind turbine, this paper used the method which combines ELMD decomposition and FCM clustering. By decomposing the fault signal by ELMD, fault feature was obtained and FCM clustering was used for fault classification. Diagnosis results of actual fault data show that the clustering accuracies of imbalance, bearing wear and gear wear were $100 \%$, which can meet the requirements of the practical of fault diagnosis of wind power gear box, and the algorithm is simple and easy to realize. The algorithm has good application value for fault diagnosis of other equipments in wind turbine.

\section{CONFLICT OF INTEREST}

The authors confirm that this article content has no conflict of interest.

Table 3. The clustering results of known samples and the classification results of unknown samples.

\begin{tabular}{|c|c|c|c|c|c|c|}
\hline & Fault Types & Sample Number & First Class Membership & Second Class Membership & Third Class Membership & Final Classification Result \\
\hline \multirow{9}{*}{$\begin{array}{l}\text { Known } \\
\text { Sample }\end{array}$} & \multirow{3}{*}{ Imbalance } & 1 & 0.0099 & 0.8955 & 0.0945 & Second class \\
\hline & & 2 & 0.0030 & 0.9723 & 0.0246 & Second class \\
\hline & & 3 & 0.0053 & 0.9482 & 0.0465 & Second class \\
\hline & \multirow{3}{*}{ Bearing Wear } & 4 & 0.9182 & 0.0374 & 0.0444 & First class \\
\hline & & 5 & 0.9949 & 0.0023 & 0.0028 & First class \\
\hline & & 6 & 0.9629 & 0.0163 & 0.0208 & First class \\
\hline & \multirow{3}{*}{ Gear Wear } & 7 & 0.0285 & 0.1374 & 0.8341 & Third class \\
\hline & & 8 & 0.0100 & 0.0598 & 0.9303 & Third class \\
\hline & & 9 & 0.0056 & 0.0590 & 0.9354 & Third class \\
\hline Unknown & (Imbalance) & 10 & 0.0183 & 0.7320 & 0.2497 & Second class \\
\hline \multirow{2}{*}{ Samples } & (Bearing Wear) & 11 & 0.9203 & 0.0354 & 0.0443 & First class \\
\hline & (Gear Wear) & 12 & 0.0085 & 0.0904 & 0.9011 & Third class \\
\hline
\end{tabular}




\section{ACKNOWLEDGEMENTS}

This paper is supported by the Fundamental Research Funds for the Central Universities(13MS102).

\section{REFERENCES}

[1] D. Berry, "Innovation and the price of wind energy in the US", Energy Policy, vol. 27, pp. 4493- 4499, 2009.

[2] H. Li, S. Guo and B. Wang, "Analysis of environmental value of wind power generation in China", Energy Technology and Economy, vol. 7, pp. 35-39, 2011.

[3] China Wind Energy Association Committee (CWEA), "2010 Chinese installed wind power capacity", Wind Energy Source, vol. 3, pp. 34-40, 2011.

[4] X. Chen, J. Li, H. Cheng, B. Li, and Z. He, "Research in wind turbine condition monitoring and fault diagnosis technology, "Chinese Journal of Mechanical Engineering, vol. 47, no. 9, pp. 45-52, 2011.

[5] Z. Hameed, Y.S. Hong, Y.M. Cho, S.H. Ahn, and C.K. Song, "Condition monitoring and fault detection of wind turbines and related algorithms: A review", Renewable and Sustainable Energy Reviews, vol. 13, no. 1, pp. 1-39, 2009.
[6] X. Liao, Z. Wan, and X. Xiong, "Roller bearing fault diagnosis method based on ELMD and LS-SVM", Journal of Chemical Industry and Engineering, vol. 12, pp. 4667-4673, 2013.

[7] J. Cheng, M. Shi, and Y. Yang, "Roller bearing fault diagnosis method based on LMD and neural network", Vibration and Shock, vol. 29, no .8, pp. 141-144, 2010.

[8] L. Zhang, H. Liao, Y. Cao, S. Luo, and Y. Zhao, "Diagnosis of engine crankshaft bearing fault based on EEMD and fuzzy $\mathrm{C}$ means clustering", Journal of Internal Combustion Engine, vol. 4, pp. 332-336, 2011.

[9] C. Zhou, X. Wu, C. Liu, and W. He, "Fault diagnosis of rolling bearing based on EMD and fuzzy C means clustering", Journal of Kunming University of Science and Technology (Science and Technology Edition), vol. 6, pp. 34-39, 2009.

[10] L. Jiang, Y. Liu, X. Li, and A. Chen, "Application of EMD fuzzy clustering method in the fault diagnosis of rolling bearing", Mechanical Strength, vol. 5, pp. 650-654, 2011.

[11] G. Li, L. Zhang, and L. Duan, "The fault diagnosis method of engine based on EMD and fuzzy clustering", Petroleum Machinery, vol. 3, pp. 49-53, 2010.

[12] W.J. Wang, and P.D. McFadden, "Application of wavelets to gear box vibration signal for Fault Detection", Journal of Sound and Vibration, vol. 192, no. 5, pp. 927-939, 1996.

[13] J. S. Smith, "The local mean decomposition and its application to EEG perception data", Journal of the Royal Society Interface, vol. 2, no.5, pp. 443-4, 2005

(C) Luo and Huang; Licensee Bentham Open

This is an open access article licensed under the terms of the Creative Commons Attribution Non-Commercial License (http://creativecommons.org/licenses/by-nc/4.0/) which permits unrestricted, non-commercial use, distribution and reproduction in any medium, provided the work is properly cited. 